\title{
PAVK: Frauen haben meist atypische Symptome
}

\section{Die American Heart Asso- ciation (AHA) publizierte kürzlich ein neues Konsen- sus-Statement zum Thema Frauen und periphere arteri- elle Verschlusskrankheit.}

- Dass die periphere arterielle Verschlusskrankheit (PAVK) bei Männern häufiger sei als bei Frauen, ist ein Mythos. Obwohl die altersabhängige Prävalenz der PAVK bei Frauen niedriger ist als bei Männern, haben insgesamt doch mehr Frauen weltweit diese Erkrankung.

Die meisten Betroffenen wissen nichts von ihrer Krankheit. Im Alter über 65 Jahren beträgt die Prävalenz der PAVK 15-25\%, und nur bei $2 \%$ der Betroffenen wurde jemals eine Messung ihres Knöchel-Arm-Index (Ankle Brachial Index $=$ ABI) durchgeführt. Die deutsche getABI-Studie zeigt nahezu identische Zahlen auch für Deutschland.

Nach wie vor wird dieses Problem sowohl von der Ärzteschaft als auch von der Bevölkerung nicht adäquat zur

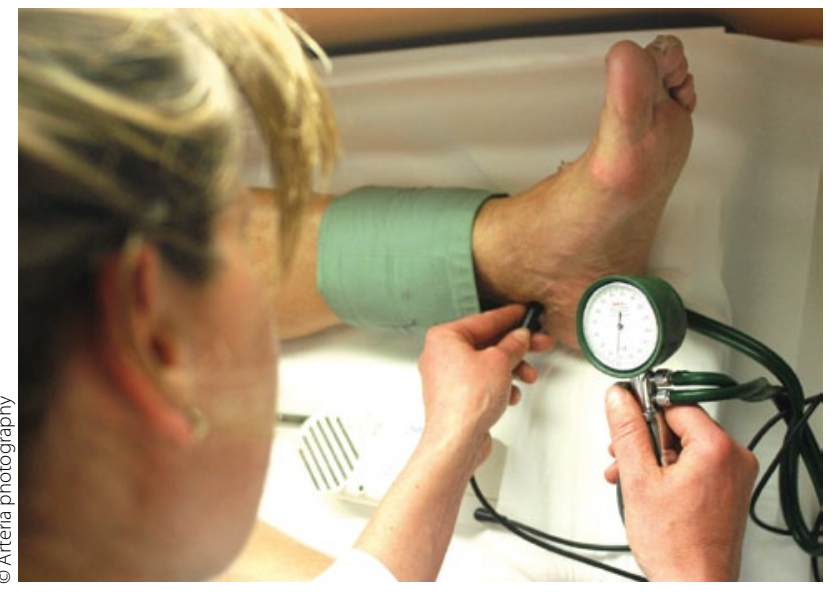

Trotz der deutlichen Prävalenz der PAVK gerade bei Frauen über 65 Jahren wird kaum jemals der Knöchel-Arm-Index bestimmt.

Kenntnis genommen. Im Gegensatz zu den Fortschritten, die bezüglich der Prävention bei der koronaren Herzerkrankung in den letzten Jahren weltweit erzielt wurden, gilt dies nicht für die PAVK. Die Prävalenz der PAVK nimmt nicht ab, die Amputationsraten steigen weiterhin kontinuierlich an.

Wie bei der koronaren Herzerkrankung haben Frauen auch deutlich mehr atypische Symptome beim Vorliegen ei- ner PAVK als Männer. Klassischerweise bekommen Patienten $\mathrm{ab}$ einer gewissen Gehstrecke krampfartige Schmerzen im Bereich der Muskulatur, vor allem in den Waden. Atypische Beschwerden sind Schmerzempfindungen im ganzen Bein, isoliert in der Ferse.

\section{Kommentar}

Für die PAVK bei Frauen gilt: Weltweit haben mehr Frauen eine PAVK als Männer. Die Komplikationsrate bei Frauen ist höher als bei Männern, wenn $\operatorname{der} A B I<0,90$ ist. Frauen (insbesondere farbige Frauen) haben häufiger Bypass-Verschlüsse und Amputationen als Männer.

Die AHA kommt zu dem Schluss, dass eine bessere "Awareness" die Grundlage für eine optimierte Erkennung und Behandlung der Erkrankung weltweit darstellt.

C. DIEHM -

- A. T. Hirsch et al.

A call to action: Women and peripheral arterial disease. A scientific from the American Heart Association. Circulation 125 (2012) 1449-1472

\section{Achtung:}

\section{Hier muss der Dummy durch eine Anzeige ersetzt werden !!}

